\title{
A NEW METHOD FOR AUTOMATIC DEFECTS DETECTION AND DIAGNOSIS IN ROLLING ELEMENT BEARINGS USING WALD TEST
}

\author{
Ammar Chiter, Rabah Zegadi, Rais El'Hadi Bekka, Ahmed Felkaoui \\ Applied Precision Mechanics Laboratory, Institute of Optics and Precision Mechanics, Ferhat Abbas University of Setif 1 , \\ Setif, Algeria; e-mail: chiter.ammar@gmail.com
}

\begin{abstract}
To detect and to diagnose, the localized defect in rolling bearings, a statistical model based on the sequential Wald test is established to generate a "hypothetical" signal which takes the state zero in absence of the defect, and the state one if a peak of resonance caused by the defect in the bearing is present. The autocorrelation of this signal allows one to reveal the periodicity of the defect and, consequently, one can establish the diagnosis by comparing the frequency of the defect with the characteristic frequencies of the bearing. The originality of this work is the use of the Wald test in the signal processing domain. Secondly, this method permits the detection without considering the level of noise and the number of observations, it can be used as a support for the Fast Fourier Transform. Finally, the simulated and experimental signals are used to show the efficiency of this method based on the Wald test.
\end{abstract}

Keywords: diagnosis, detection, rolling element bearing, defect, Wald sequential test

\section{Introduction}

In the industry, a great attention has been given to monitoring conditions and maintenance for the purpose to improve the quality of production. Edwards et al. (1998) and Tandon and Choudhury (1999) showed the importance of maintenance as the best way to avoid maintenance problems that are often very expensive. And also how the predictive maintenance techniques have evolved to keep a check of mechanical health by generating information on the machine condition. In rotating machines, the transmission elements: belts, gears and bearings are of major interest in industrial maintenance as the operation of a mechanical system heavily depends on health of these elements. Particularly, the rolling bearing is one of the most critical components that determine machinery health and its remaining life time in modern production machinery (Jayaswal et al., 2008). Robust Predictive Health Monitoring tools are needed to guarantee the healthy state of rolling bearings during the operation. A predictive health monitoring tool indicates upcoming failures which provide sufficient lead time for maintenance planning, as showed by El-Thalji and Jantunen (2015), Mann et al. (1995) and Renwick and Babson (1985).

Over the past two decades, several methods have been the subject of studies and developments. Visibly noticed are revolution methods based on mechanical signal processing, which are divided into two main categories, detection and diagnosis, and are based on time-frequency methods and temporal methods or a combination of both. Thus, many methods are born, the scalar indicators such as kurtosis, skew, crest factor (Dron et al., 2004; Pachaud et al., 1997), demodulation and detection of the envelope (Sheen, 2004, 2008), amplitude modulation (Stack et al., 2004), detection of vibration modes (Rizos et al., 1990), de-noising vibratory signals (Bolaers et al., 2004), the spectral density analysis (Krejcar and Frischer, 2011), the Fast Fourier Transform (Lenort, 1995), the statistical model based on hypothesis test as KS-test Kolmogorov and Smirnov (Kar and Mohanty, 2004; Dong et al., 2011; Yang et al., 2005), scalar and vector statistical time series methods (Kopsaftopoulos and Fassois, 2011), neural networks (Samanta 
and Al-Balushi, 2003), wavelets (Bendjama and Boucherit, 2016), blind source separation (Wang et al., 2014), fuzzy logic (Liu et al., 1996). El-Thalji and Jantunen (2015) and Rai and Upadhyay (2016) reviewed almost all the techniques used in the domain predicting defects.

Typical defects in bearings are localized defects that occur generally in form of tiredness cracking under cyclic pressure of contact (El-Thalji and Jantunen, 2015; Fajdiga and Sraml, 2009; Glaeser and Shaffer, 1996; Ismail et al., 1990; Tauqir et al., 2000). Thus, the detection of cracking is frequently based on detection of the attack. During an abnormal operation, a series of wide band impulses will be generated when the rolling element of the bearing (ball or roller) (Brie, 2000; Ou et al., 2016) goes above the defect at a frequency determined by the shaft speed, geometry of the bearing and the site of the defect (Barkov, 1999; Dyer and Stewart, 1978; Feng et al., 2016; Ma and Li, 1995; Tandon and Choudhury, 1999). The site of the defect depending on the characteristic frequencies gives the possibility of detecting the presence of the defect and performing the diagnosis of the defective part.

The difficulty of detection of localized defects (Niu et al., 2015) is related to the bearing energy which will diffuse through a wide band of frequency and hence it can be easily immersed in the noise (Ma and Li, 1995; Van et al., 2016). Thus, under various operating regimes (varying loads and speeds), many methods remain inefficient for the prediction (El-Thalji and Jantunen, 2015), because it may happen that an excited resonance mode at the beginning of the attack may not be excited later when the defect has developed (Ma and Li, 1995; Mikhlin and Mytrokhin, 2008). In this paper, and to refer on the sequential analysis developed by Wald in the 1940s (Schneeweiss, 2005; Wald, 1943, 1945, 1947, 1949; Wald and Wolfowitz, 1943, 1948), a composite hypothesis test is used for the detection and diagnosis of localized defects in rolling bearings. To this end, it is necessary to be provided with a significant and exact variance without any need to estimate when the resonances modes occur.

\section{Problem position}

\subsection{Probability Density Function (PDF) of vibrations of rolling bearings}

To characterize vibration of rolling bearings, which is supposed to be a stationary stochastic process, and the PDF can describe the percentage in time when the signal reaches a given amplitude $x$. For the given amplitude, the PDF is estimated by

$$
P(x)=\lim _{\Delta x \rightarrow 0} \frac{P_{r}\{x \leqslant x(t) \leqslant x+\Delta x\}}{\Delta x}=\lim _{\Delta x \rightarrow 0} \frac{1}{\Delta x} \sum_{i=1}^{j} \frac{\Delta t_{i}}{T}
$$

where $T$ is the total time of observation and $\Delta t_{i}$ is the $i$-th duration while $x(t)$ is inside the interval $[x, x+\Delta x]$. For vibration without a defect, which represents healthy functioning, the distribution of the amplitude can be considered as a Gaussian process. This vibratory signature will have a well-defined variance $\sigma_{0}^{2}$ which is different from the variance $\sigma_{1}^{2}$ of a signal with a localized fault (Fig. 1) and, consequently, the overall vibration of the bearing will be constituted by two alternately periodic parts with different variances (Ma and Li, 1995).

\subsection{Sequential Probability Test (SPRT)}

Introduce now the sequential probability test (SPRT) of a simple null hypothesis $H_{0}$ which indicates the good operating condition and a simple alternative hypothesis $H_{1}$ which indicates the presence of a defect, based on $N$ independent observations $x_{1}, x_{2}, \ldots, x_{N}$ having a common probability density function developed by Wald $(1945,1949)$ and Weiss $(1956)$. 


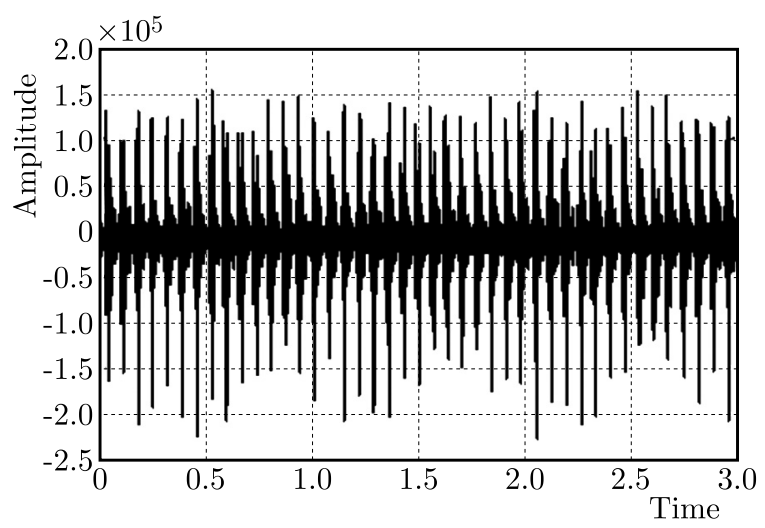

Fig. 1. Real signal of the rolling bearing with a defect

The hypotheses are

$$
\begin{aligned}
& H_{0}: \quad P\left(\mathbf{x} / H_{0}\right)=\frac{1}{\sqrt{(2 \pi)^{N}} \sigma_{0}^{N}} \exp \left(-\sum_{i=1}^{N} \frac{x_{i}^{2}}{2 \sigma_{0}^{2}}\right) \\
& H_{1}: \quad P\left(\mathbf{x} / H_{1}\right)=\frac{1}{\sqrt{(2 \pi)^{N}} \sigma_{1}^{N}} \exp \left(-\sum_{i=1}^{N} \frac{x_{i}^{2}}{2 \sigma_{1}^{2}}\right)
\end{aligned}
$$

where $\mathbf{x}=\left[x_{1}, x_{2}, \ldots, x_{N}\right]$ and $\sigma_{i}^{2}$ are the variances with $\sigma_{0}^{2}<\sigma_{1}^{2}$.

For the analysis of any vibratory signal, certainly one of the two variances will be retained outside the test hypothesis $H_{1}$ and one will have information whether or not it occurs with one of the characteristic frequencies of the rolling bearings (inner race, outer race, ball and cage). In the case of healthy rolling bearings, during a time $\Delta t$ for the signal $x(t)$, all measurements of $M$ observations will have a Gaussian distribution given by relation $(2.2)_{1}$, In the case of a defective bearing given by $(2.2)_{2}$ and by varying the number $M$ of observations in the time $\Delta t$, and as soon as $M$ is sufficiently large, and it is always possible to calculate the estimated variance $\sigma_{1}^{2}$ of the acquired vibratory signal with the defect in the rolling bearing. The variances $\sigma_{0}^{2}$ for healthy rolling bearings could be calculated by

$$
\sigma_{0}^{2}=\frac{1}{M} \sum_{1}^{M} x_{i}^{2}
$$

and $\sigma_{1}^{2}=M^{-1} \sum_{1}^{M} x_{i}^{2}$ is considered as an estimated variance of the defect signal. Such an estimate will lead to a test for probability of both detection or false alarm (Ma and Li, 1995).

\section{Sequential test}

\subsection{The likelihood ratio test with simple choice}

The likelihood ratio test (PRT) of the $\sigma_{1}^{2}$ measurement could then be expressed as follows (Ma and Li, 1995; Paulson, 1947)

$$
\left\{\begin{array}{lll}
\text { if } & \xi(\mathbf{x})>\mu & \text { choose } H_{1} \\
\text { if } & \xi(\mathbf{x})<\mu & \text { choose } H_{0}
\end{array}\right.
$$

where $\xi(\mathbf{x})$ is the likelihood ratio, which is defined by

$$
\xi(\mathbf{x})=\frac{P\left(\mathbf{x} / H_{1}\right)}{P\left(\mathbf{x} / H_{0}\right)}=\frac{\sigma_{1}^{N}}{\sigma_{0}^{N}} \exp \left(\frac{\sigma_{1}^{2}-\sigma_{0}^{2}}{2 \sigma_{0}^{2} \sigma_{1}^{2}} \sum_{i=1}^{N} x_{i}^{2}\right)
$$


By taking the natural logarithm of the two parts, the test can be simplified into

$$
\left\{\begin{array}{lll}
\text { if } & f(\mathbf{x})>\gamma & \text { choose } H_{1} \\
\text { if } & f(\mathbf{x})<\gamma & \text { choose } H_{0}
\end{array}\right.
$$

where

$$
f(\mathbf{x})=\sum_{i=1}^{N} x_{i}^{2} \quad \gamma=\frac{2 \sigma_{0}^{2} \sigma_{1}^{2}}{\sigma_{1}^{2}-\sigma_{0}^{2}} \ln \left(\frac{\sigma_{1}^{N}}{\sigma_{0}^{N}} \mu\right)
$$

Then probability $P_{f}$ of the false alarm and the detection probability $P_{d}$ of the PRT are

$$
\begin{gathered}
P_{f}=P\left(f(\mathbf{x})>\gamma / H_{0}\right)=P\left(\sum x_{i}^{2}>\gamma / H_{0}\right)=\int_{\sum x_{i}^{2}>\gamma} P\left(\mathbf{x} / H_{0}\right) d \mathbf{x} \\
P_{d}=P\left(f(\mathbf{x})>\gamma / H_{1}\right)=P\left(\sum x_{i}^{2}>\gamma / H_{1}\right)=\int_{\sum x_{i}^{2}>\gamma} P\left(\mathbf{x} / H_{1}\right) d \mathbf{x}
\end{gathered}
$$

From equations $(2.2)$ and $(3.5)_{1}$, the $P_{f}$ is a decreasing monotone function of the parameter $\gamma$. Integration of equations (3.5) leads to

$$
P_{f}=\exp \left(\frac{-\gamma}{2 \sigma_{0}^{2}}\right) \quad P_{d}=\exp \left(\frac{-\gamma}{2 \sigma_{1}^{2}}\right)
$$

where $\sigma_{0}^{2}$ is the variance measured during healthy operation, $\sigma_{1}^{2}$-variance measured during unspecified operation and $\gamma$ - the threshold of the test determined by

$$
\gamma=-2 \sigma_{0}^{2} \ln P_{f}
$$

while combining $P_{d}$ with $P_{f}$ we will have

$$
P_{d}=P_{f}^{\frac{\sigma_{0}^{2}}{\sigma_{1}^{2}}}
$$

Using equation $(3.5)_{1}$ to determine the probability of the false alarm $P_{f}$ which corresponds to threshold equation (3.7) on the one hand and, on the other, using this same threshold will give the maximum probability of detection $P_{d}$ defined by equation $(3.5)_{2}$ related to the variance $\sigma_{1}^{2}$ which is an unknown parameter estimated in one duration of the previously signal fixed. It can be deduced that the uniformly most powerful test (UMP) exists in the sense of Neyman-Pearson criterion which maximizes $P_{d}(3.8)$ for a given $P_{f}$ because the optimal probability rate test (PRT) (3.3) for each $\sigma_{1}^{2}>\sigma_{0}^{2}$ could be completely defined apart from the knowledge of the true variance $\sigma_{1}^{2}$ of the signal defect. Finally, the UMP test is defined by system (3.3) and is constructed by equations (3.1), (3.2) with a determined $\gamma$ by the pre-established false alarm probability $\alpha$, where $\alpha$ is the threshold of significance

$$
P_{f}(\gamma)=\alpha
$$

\subsection{Wald sequential test}

Contrarily to the classical test (test with a simple choice), one is not obliged to make a choice between the two hypotheses $H_{0}$ and $H_{1}$, consequently, one deals with another type of test. If the size of observations is fixed, the construction of the test leads to the sharing of possible values 
of the statistical domain in three regions (Wald, 1945; Berger and Wald, 1949; Wolfowitz, 1949; Sobel and Wald, 1949)

$$
\Psi^{(n)}=\Psi\left(x_{1}, x_{2}, \ldots, x_{n}\right)
$$

that is the region of probable values and the region of improbable values (knowing that the basic hypothesis $H_{0}$ is true). If a given value of $\Psi\left(x_{1}, x_{2}, \ldots, x_{n}\right)$ falls into the region of improbable values, the basic hypothesis is rejected. The sequential test, that is, the test based on a sequential procedure of observation, is built up as follows. For each value of

$$
\nu=1,2, \ldots, n, n+1
$$

the domain $\Gamma_{\nu}$ of possible values of the critical statistics $\Psi\left(x_{1}, x_{2}, \ldots, x_{n}\right)$ is divided into three disjoined regions: $\Gamma_{\nu}^{H_{0}}$ - region of probable values, $\Gamma_{\nu}^{H_{1}}$ - region of improbable values and $\Gamma_{\nu}^{*}-$ region of doubtful values (knowing that $H_{0}$ is true)

$$
\Gamma_{\nu}=\Gamma_{\nu}^{H_{0}} \cup \Gamma_{\nu}^{H_{1}} \cup \Gamma_{\nu}^{*}
$$

where $\nu=1,2, \ldots$ with each step $\nu$ of the sequential procedure of observation. After having recorded the observations $x_{1}, \ldots, x_{\nu}, \nu=1,2, \ldots$ one makes a decision relying on the following rule which defines the Wald test: if $\Psi\left(x_{1}, x_{2}, \ldots, x_{n}\right) \in \Gamma_{\nu}^{H_{0}}$ one accepts $H_{0}$; if $\Psi\left(x_{1}, x_{2}, \ldots, x_{n}\right) \in$ $\Gamma_{\nu}^{H_{1}}$ one accepts $H_{1}$ and if $\Psi\left(x_{1}, x_{2}, \ldots, x_{n}\right) \in \Gamma_{\nu}^{*}$ the problem remains open until the $\nu$-th observation. For this reason, the region $\Gamma_{\nu}^{*}$ is called the region of indetermination or the region of the observation pursuit.

For the establishment of the Wald test of probability, one considers two simple hypotheses of the following form, see Wald $(1945,1947)$ and Wald and Wolfowitz (1948)

$H_{0} \quad$ The observation is extracted from a density population $f\left(x, \theta_{0}\right)$

$H_{1} \quad$ The observation is extracted from a density population $f\left(x, \theta_{1}\right)$

The critical statistics of this test is defined by the relation (Wald, 1945, 1947; Paulson, 1947)

$$
\Psi^{(\nu)}=\ln \frac{f\left(x_{1}, \theta_{1}\right) \cdots f\left(x_{i}, \theta_{1}\right)}{f\left(x_{1}, \theta_{0}\right) \cdots f\left(x_{i}, \theta_{0}\right)}=\sum_{i=1}^{\nu} \ln \frac{f\left(x_{i}, \theta_{1}\right)}{f\left(x_{i}, \theta_{0}\right)}
$$

where: $f\left(x_{i}, \theta_{0}\right)=P\left(\mathbf{x} / H_{0}\right)$ with $\theta_{0}=\sigma_{0}^{2}$ and $f\left(x_{i}, \theta_{1}\right)=P\left(\mathbf{x} / H_{1}\right)$ with $\theta_{1}=\sigma_{1}^{2}$.

$P\left(\mathbf{x} / H_{0}\right)$ and $P\left(\mathbf{x} / H_{1}\right)$ could be drawn from equation (2.2). So, one establishing the likelihood ratio, the critical statistics would be expressed as follows

$$
\Psi^{(\nu)}=\ln \left\{\left[\frac{\exp \left(-\frac{1}{2 \sigma_{1}^{2}} \sum_{i=1}^{\nu} x_{i}^{2}\right)}{\sqrt{(2 \pi)^{\nu}} \sigma_{1}^{\nu}}\right] /\left[\frac{\exp \left(-\frac{1}{2 \sigma_{0}^{2}} \sum_{i=1}^{\nu} x_{i}^{2}\right)}{\sqrt{(2 \pi)^{\nu}} \sigma_{0}^{\nu}}\right]\right\}
$$

After simplification of equation (3.15) and arrangement of the logarithmic term, one gets

$$
\Psi^{(\nu)}=\frac{\sigma_{1}^{2}-\sigma_{0}^{2}}{2 \sigma_{0}^{2} \sigma_{1}^{2}} \sum_{i=1}^{\nu} x_{i}^{2}+\frac{\nu}{2} \ln \frac{\sigma_{1}^{2}}{\sigma_{0}^{2}}
$$

The three regions are defined roughly by relations (3.11), (3.12), (3.13) and (3.16) that define the completely Wald test (WT) (Aïvazian, 1986; Wald, 1947; Weiss, 1956)

$$
\begin{aligned}
& \Gamma_{\nu}^{H_{0}}=\left\{\Psi: \Psi^{(\nu)} \leqslant \ln \frac{\beta}{1-\alpha}\right\} \quad \Gamma_{\nu}^{H_{1}}=\left\{\Psi: \Psi^{(\nu)} \leqslant \ln \frac{1-\beta}{\alpha}\right\} \\
& \Gamma_{\nu}^{*}=\left\{\Psi: \ln \frac{\beta}{1-\alpha} \leqslant \Psi^{(\nu)} \leqslant \ln \frac{1-\beta}{\alpha}\right\}
\end{aligned}
$$

Wald test (3.17) is more optimal than all tests between hypotheses (3.13) with risks of the first and second species lower than the respective given values $\alpha$ and $\beta$.

Values of $\alpha$ and $\beta$ (Aïvazian, 1986) are: 0.1, 0.05, 0.025, 0.01, 0.005, 0.001, 0.002 . 


\section{Rolling element bearings defects detection}

\subsection{Detection procedure}

The detection procedure is divided into many steps which can be stated as follows:

1 - Take the discrete vibration for $M$ samples, which is larger than the amount of the characteristic period of the defect.

2 - Select a window of size $N$ for the test.

3 - Estimate the variance $\sigma_{0}^{2}$ by using equation (2.3).

4 - Suggest a choice of $\alpha$ and $\beta$.

5 - Position the window at the beginning of recording of the vibration.

6 - Compute $\Psi^{(N)}$ by using equation (3.16).

7 - Define the intervals of the three regions by the terminals $a=\ln [\beta /(1-\alpha)]$ and $b=\ln [(1-\beta) / \alpha]$.

8 - Make the test by using equation (3.17).

9 - Generate a hypothetical signal defined by

$$
h(i)=\left\{\begin{array}{lllll}
0 & \text { if } & H_{0} & \text { is true } & (\Psi \leqslant a) \\
1 & \text { if } & H_{1} & \text { is true } & (\Psi \geqslant b)
\end{array}\right.
$$

If $a \leqslant \Psi \leqslant b$, carry on with pursue for data opening another window (here, one does not make a decision but only increases the size of the window).

10 - After generation of the hypothetical signal, if a defect is present, there will be a data vector composed of two values 0 and 1 . If 1 , then appears periodically with a period of the characteristic frequency of the bearing and is considered defective.

11 - To compare the detected frequency with the main characteristic frequencies of the rolling bearings, it would be very easy to locate the defect so the diagnosis could be established by comparing the multiple of this frequency detected with that of the most well-known defects.

\subsection{Test plan}

Based on the detection procedure described in Section 4.1, a test plan can be established which is shown by the procedure diagram shown in Fig. 2. So that the experiment is valid, one chooses $N$ as a small fraction of the characteristic period of the defect, that is to say one fifth (Ma and Li, 1995). By examining step 10 in the detection procedure in Section 4.1 (to show if there is periodicity), one uses autocorrelation of the signal, a peak in the autocorrelation function reveals the periodicity of the signal, and the value of the time of this peak will give the period of the defect $T_{d}$. Consequently, one can determine the frequency of the defect $f_{d}$, and comparing it with the characteristic frequency $f_{c}$, one can establish the diagnosis.

\section{Validation of the model by simulated and experimental signals}

\subsection{Validation of the model by simulated signals}

\subsubsection{Generation of the simulated signals}

To simulate the defect, a bearing of the type NJ2204ECP has been used. The shaft speed is $n=1500 \mathrm{rpm}$, the characteristic frequencies are determined by the relations from Appendix A1, 


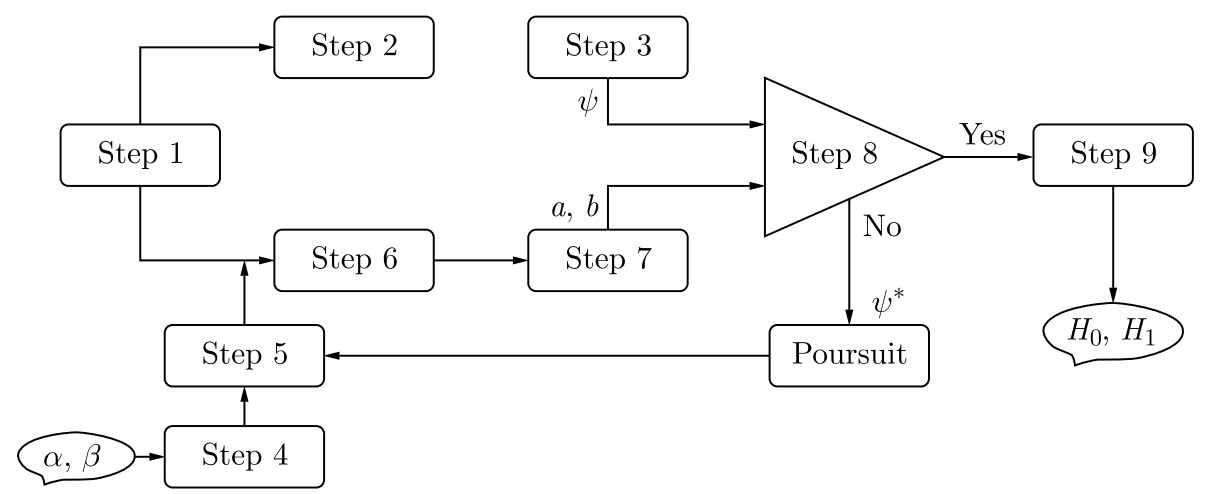

Fig. 2. Test plan

where the frequency of the cage is: $f_{\text {cage }}=0.39 f r(9.74 \mathrm{~Hz})$, the frequency of the outer race: $f_{\text {or }}=0.39 Z \mathrm{fr}(87.68 \mathrm{~Hz})$, the frequency of the inner race $f_{i r}=0.61 Z$ fr $(137.32 \mathrm{~Hz})$, and the frequency of the ball: $f_{r e}=4.754 f r(118.85 \mathrm{~Hz})$; where: $f r=25 \mathrm{~Hz}, Z$ is the number of balls. For NJ2204ECP: $Z=9, d=7.5 \mathrm{~mm}, D=34 \mathrm{~mm}, \alpha=0$. The reference signal (Fig. 3a) is taken as a sinusoid of frequency $25 \mathrm{~Hz}$, amplitude equal to unit and a null phase. The simulated defect signal (Fig. 3b) is considered as the sum of a sinusoid of frequency $25 \mathrm{~Hz}$, amplitude equal to unit and the null phase, a sinusoid of frequency $87 \mathrm{~Hz}$ of amplitude 10 times the unit (representing a defect of frequency $87 \mathrm{~Hz}$, which corresponds to the frequency of the outer race, as one can use the function pulstran available in Matlab which generates a series of impulses), and a Gaussian white noise centered with variance equal to 1 generated by the function "randn" available in Matlab with a signal noise ratio SNR $=20 \mathrm{~dB}$. The thresholds of significances are fixed at $\alpha=0.05$ and $\beta=0.002$.
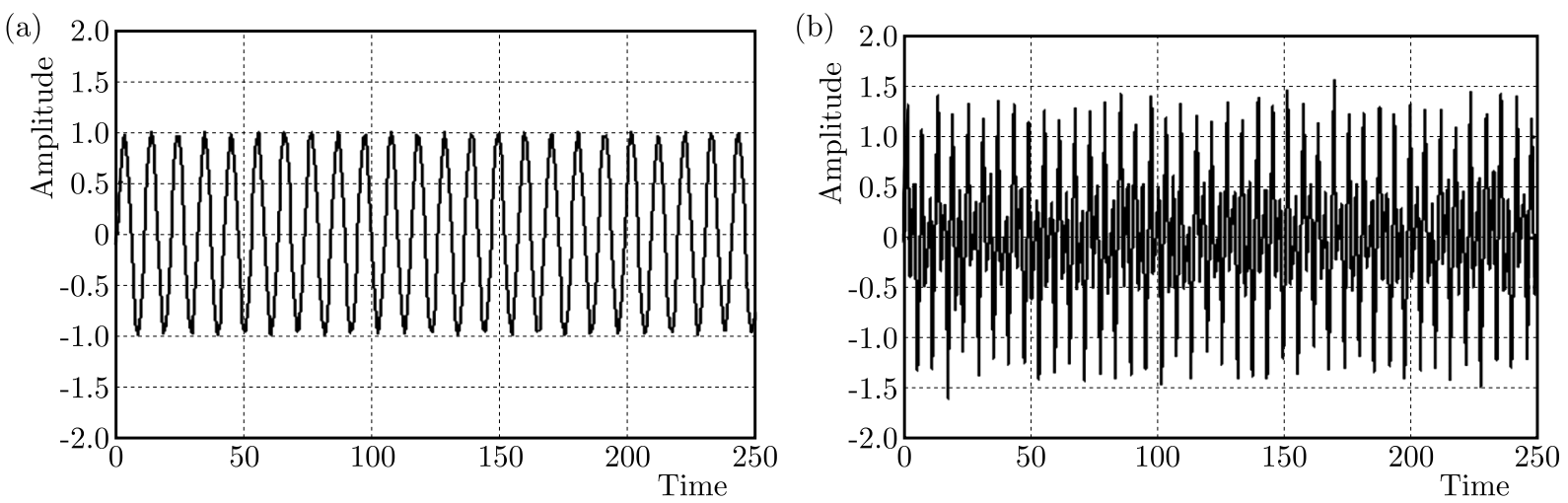

Fig. 3. (a) Reference signal, (b) defect signal

\subsubsection{Interpretation}

One can say that periodicity of a hypothetical signal ( $h$-signal, Fig. 4a) appeared in the function of autocorrelation (Fig. 4b) reveals the existence of a defect. To determine its frequency, one carries out Fourier fast transform (FFT) of the hypothetical signal, which reveals visually the frequency of the defect $(87 \mathrm{~Hz})$ which corresponds indeed to the characteristic frequency of the outer race (Fig. 4c). Consequently, one can affirm that the plan suggested for detection and diagnosis of the defect in the bearing has succeeded and to made diagnosis of the defective part. During healthy running, the hypothetical signal will be zero, the autocorrelation of the $h$-signal will not reveal any periodicity, and the FFT will confirm the absence of the defect. 
(a)

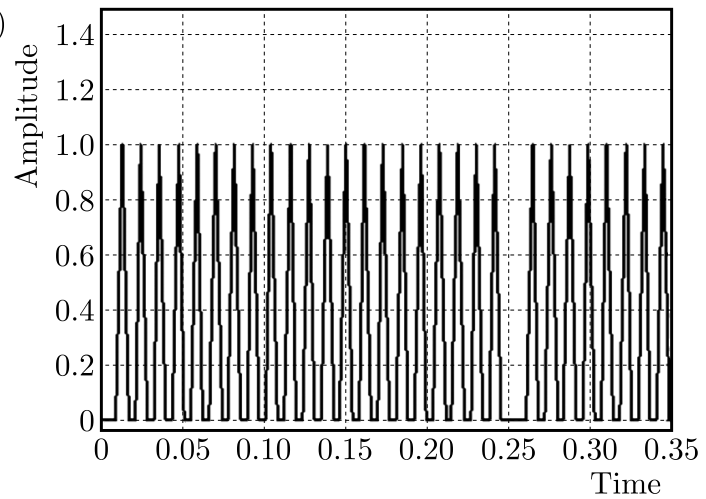

(b)

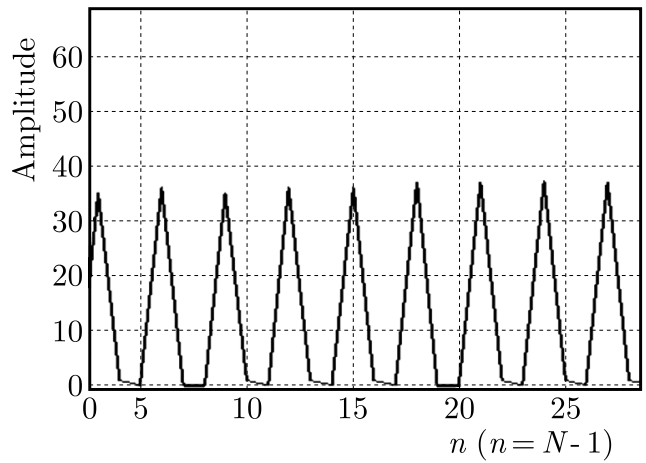

(c)

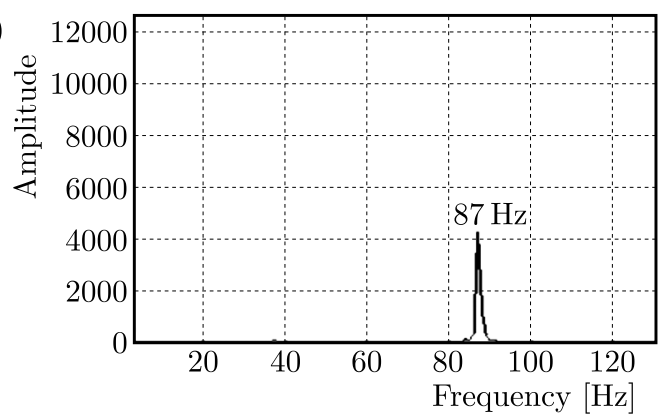

Fig. 4. (a) Hypothetical signal, (b) autocorrelation of hypothetical signal, (c) FFT of hypothetical signal

\subsection{Validation of the model by experimental signals}

\subsubsection{Generation of the experimental signals}

The test stand consists of a reinforced concrete frame, isolated from the ground by shockabsorbing studs. Two rows of shafts each having diameter of $60 \mathrm{~mm}$ and length of $680 \mathrm{~mm}$ are mounted in an open loop and fixed to the chassis by four rolling bearings with an average stiffness of $3 \cdot 10^{7} \mathrm{daN} / \mathrm{m}$ as shown in Fig. 5 .
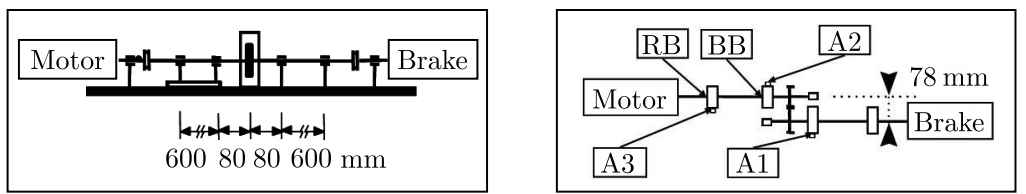

Fig. 5. Architecture of the teststand (RB - roller bearing, BB - ball bearing)

The bearings in the vicinity of the test gear pair have ball bearings of the type 6012, while the outer bearings are roller bearings of the type NU1013. The shaft lines are connected in rotation by test gears. The applied speed and torque are measured by an electronic device composed of a motor and a brake.

The dynamic behavior of the system can be studied using measurements of the acceleration, transmission error and noise. The accelerations are measured using piezoelectric accelerometers ENDEVCO $224 \mathrm{C}$ whose resonance frequency is $32 \mathrm{kHz}$. The accelerometers are mounted by gluing small duralumin pellets onto the accelerometers which are screwed. The tests are carried out on a spur gear with helical teeth. The gear ratio is $36 / 38$ with modulus $m=2$. The geometric characteristics of the ball and roller bearing are given in Table 1.

Type of defect: To simulate the scaling on the bearings, a notch of $1.7 \mathrm{~mm}$ and depth of $0.088 \mathrm{~mm}$ is made using a fine grinder as shown in Fig. 6. The roller bearing is removable without "NU type" destruction or specialized tooling. 
Table 1. Geometric characteristics of the ball and roller bearing

\begin{tabular}{|l|c|c|}
\hline \multicolumn{1}{|c|}{ Geometric characteristics } & Ball bearing & Roller bearing \\
\hline \hline Middle diameter to center of balls $D[\mathrm{~mm}]$ & 77.7 & 80.55 \\
\hline Diameter of ball $d[\mathrm{~mm}]$ & 9 & 7 \\
\hline Number of balls $Z$ & 14 & 21 \\
\hline Angle of contact $\alpha$ & 0 & 0 \\
\hline
\end{tabular}

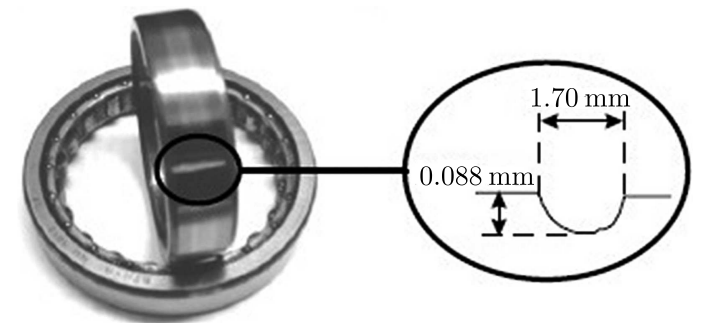

Fig. 6. Defective inner race geometry of the roller bearing

Monitoring conditions: The applied load is equal to $12 \mathrm{daN} \mathrm{m}$ and $4300 \mathrm{rpm}$ speed test. The characteristic frequencies of the ball bearing and the roller bearing are calculated by the geometrical formulas given in Appendix A1.

Table 2. Characteristic frequencies of the ball and roller bearings

\begin{tabular}{|l|c|c|c|c|c|}
\hline Bearing type & $F_{r}[\mathrm{~Hz}]$ & $F_{\text {cage }}[\mathrm{Hz}]$ & $F_{\text {or }}[\mathrm{Hz}]$ & $F_{\text {ir }}[\mathrm{Hz}]$ & $F_{\text {er }}[\mathrm{Hz}]$ \\
\hline \hline 6012 & 71.67 & 31.68 & 443.56 & 559.77 & 627.02 \\
\hline NU1013 & 71.67 & 32.72 & 687.11 & 817.89 & 830.91 \\
\hline
\end{tabular}

$F_{r}$ - rotating frequency, $F_{\text {cage }}$ - frequency of the cage,

$F_{o r}$ - frequency of the outer race, $F_{i r}$ - frequency of the inner race,

$F_{e r}$ - frequency of the ball or roller

Experimental signals: The acquired reference signal and the acquired signal defect are shown in Fig. $7 \mathrm{a}$ and $7 \mathrm{~b}$.

\subsubsection{Interpretation}

The detection and diagnostic plan applied to the experimental signals shown in Fig. 7a and Fig. $7 \mathrm{~b}$ is able to detect the fault frequency applied to the bearing inner ring shown in Fig. 8a. It shows the presence of state "1" of the hypothetical signal and Fig. $8 \mathrm{~b}$ shows a frequency of $814 \mathrm{~Hz}$ very close to the fault frequency which is equal to $817.89 \mathrm{~Hz}$. It indicates that the plan has reacted well in establishing a correct diagnosis.

\section{Diagnosis plan}

To establish a good diagnosis of defects, it is necessary to know a significant number of defects. Thus, by comparing the frequency detected by the Wald test presented before with the characteristic frequencies we can locate the defect. By comparing the defect frequency with the main defects of the rolling bearings (Barkov, 1999), we can establish the diagnosis by using the frequency of modulation presented in the work of Barkov (1999). For the plan suggested by Fig. 2 it is possible to establish the diagnosis of the bearing defective part and its nature. 

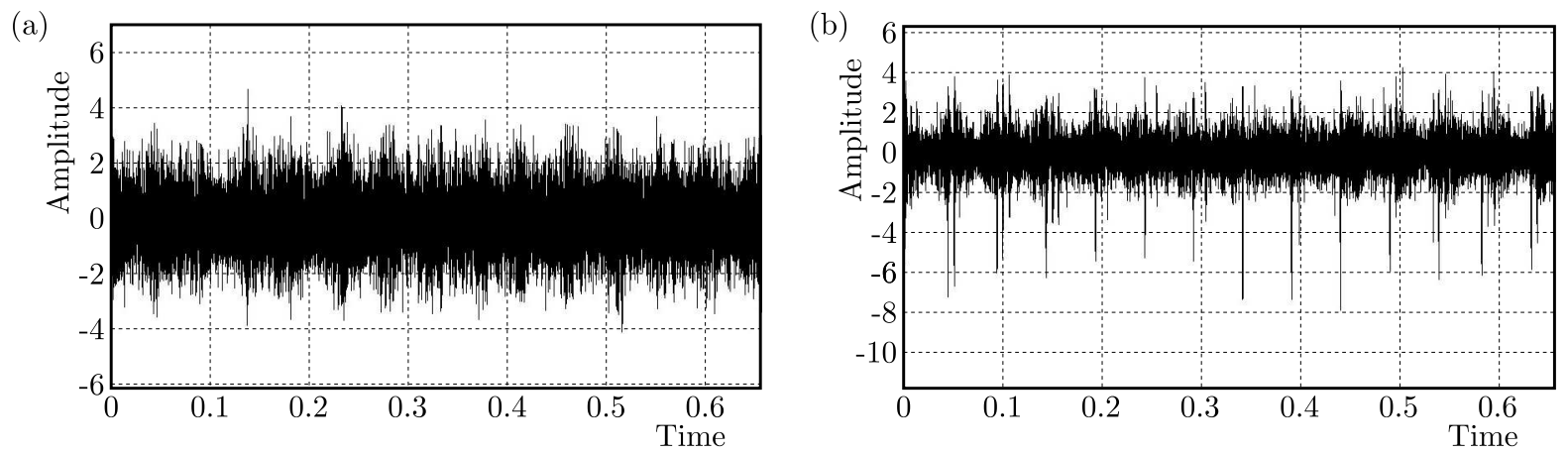

Fig. 7. (a) Experimental reference signal, (b) experimental defect signal

(a)

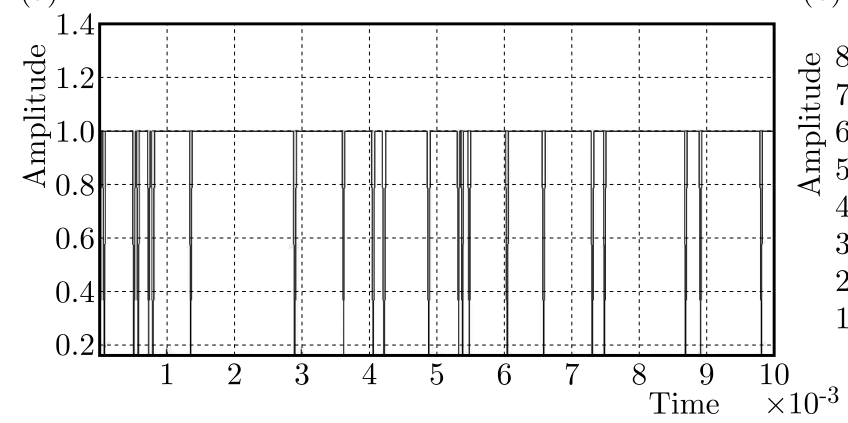

(b)

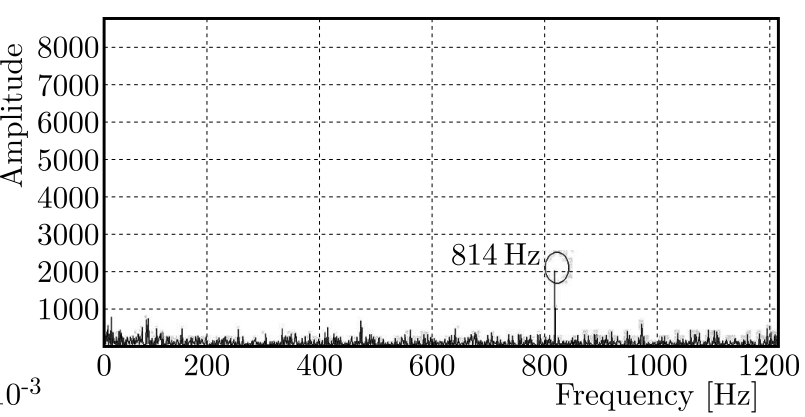

Fig. 8. (a) Experimental hypothetical signal, (b) FFT of the experimental hypothetical signal

\section{Conclusion}

The detection and diagnosis plan based on the Wald test is described. This plan can be applied to measurements of the bearings vibration signals with and without defects under various loads and speeds. The effectiveness of the suggested detection plan is illustrated in Fig. 4 for the simulated signal and in Fig. 8 for the experimental signal. The plan works very well with vibratory signals of wide bands. Finally, the plan is very promising for automatic detection and diagnostic applications.

\section{Acknowledgment}

I address all my gratitude to the Dynamics and Control of Structure team of the Contacts and Structures Mechanics Laboratory (LaMCoS), research unit (UMR5259) of INSA Lyon France and CNRS (INSIS, Institute of Engineering Sciences and Systems) for providing me with the experimental signals realized on their own test stands and, particularly, Associate Professor Mahfoud Jarir, also the director of LaMCoS laboratory.

\section{Appendix 1}

Characteristic frequencies of the bearing (Barkov, 1999):

- frequency of the cage

$$
f_{\text {cage }}=\frac{f_{r}}{2}\left(1-\frac{d}{D} \cos \alpha\right)
$$

- frequency of the outer race

$$
f_{o r}=Z \frac{f_{r}}{2}\left(1-\frac{d}{D} \cos \alpha\right)
$$


— frequency of the inner race

$$
f_{i r}=Z \frac{f_{r}}{2}\left(1+\frac{d}{D} \cos \alpha\right)
$$

— frequency of the ball

$$
f_{r e}=f_{r} \frac{d}{D}\left[1+\left(\frac{D}{d}\right)^{2} \cos \alpha\right]
$$

where $\alpha$ is the angle of contact, $d[\mathrm{~mm}]$ - diameter of the ball, $D[\mathrm{~mm}]$ - middle distance to the center of balls, $Z$ - number of balls, $f_{r}[\mathrm{~Hz}]$ - rotating frequency $\left(f_{r}=n / 60\right), n[\mathrm{rpm}]-$ shaft speed.

\section{References}

1. Aïvazian S., Énukov I., Méchalkine, 1986, Modeling Element and Primary Data Processing (in French), Mir Moscou

2. Barkov A.V., Barkova N.A., Yudin I.A., Rogov S.N., 1999, The Peculiarities of Rolling Element Bearing Vibroacoustic Diagnostics for Transportation Applications, Vibrotek

3. Bendjama H., Boucherit M.S., 2016, Wavelets and principal component analysis method for vibration monitoring of rotating machinery, Journal of Theoretical and Applied Mechanics, 54, 2, $659-670$

4. Berger A., Wald A., 1949, On distinct hypotheses, The Annals of Mathematical Statistics, 20, $1,104-109$

5. Bolaers F., Cousinard O., Marconnet P., Rasolofondraibe L., 2004, Advanced detection of rolling bearing spalling from de-noising vibratory signals, Control Engineering Practice, 12, $181-190$

6. BRIE D., 2000, Modelling of the spalled rolling element bearing vibration signal : An overview and some results, Mechanical Systems and Signal Processing, 14, 3, 353-369

7. Dong Y., Liao M., Zhang X., Wang F., 2011, Faults diagnosis of rolling element bearings based on modified morphological method, Mechanical Systems and Signal Processing, 25, 1276-1286

8. Dron J.P., Bolaers F., Rasolofondraibe L., 2004, Improvement of the sensitivity of the scalar indicators (crest factor, kurtosis) using a de-noising method by spectral subtraction: application to the detection of defects in ball bearings, Journal of Sound and Vibration, 270, 270, 61-73

9. Dyer D., Stewart R.M., 1978, Detection of rolling element bearing damage by statistical vibration analysis, Journal of Mechanical Design, 100, 229

10. Edwards D.J., Holt G.D., Harris F.C., 1998, Predictive maintenance techniques and their relevance to construction plant, Journal of Quality in Maintenance Engineering, 4, 1, 25-37

11. El-Thalji I., JAntunen E., 2015, A summary of fault modelling and predictive health monitoring of rolling element bearings, Mechanical Systems and Signal Processing, 60-61, 252-272

12. Fajdiga G., Sraml M., 2009, Fatigue crack initiation and propagation under cyclic contact loading, Engineering Fracture Mechanics, 76 , 1320-1335

13. Feng Z., Ma H., Zuo M.J., 2016, Vibration signal models for fault diagnosis of planet bearings, Journal of Sound and Vibration, 370, 372-393

14. Glaeser W.A., Shaffer S.J., 1996, Contact fatigue, ASM Handbook, Fatigue and Fracture, 19, $331-336$

15. Ismail F., Ibrahim A., Martin H.R., 1990, Identification of fatigue cracks from vibration testing, Journal of Sound and Vibration, 140, 2, 305-317 
16. Jayaswal P., Wadhwani A.K., Mulchandani K.B., 2008, Machine fault signature analysis, International Journal of Rotating Machinery, DOI: 10.1155/2008/583982

17. Kar C., Mohanty A.R., 2004, Application of KS test in ball bearing fault diagnosis, Journal of Sound and Vibration, 269, 439-454

18. Kopsaftopoulos F.P., FAssois S.D., 2011, Scalar and vector time series methods for vibration based damage diagnosis in a scale aircraft skeleton structure, Journal of Theoretical and Applied Mechanics, 49, 3, 727-756

19. Krejcar O., Frischer R., 2011, Non destructive defect detection by spectral density analysis, Sensors, 11, 2334-2346

20. Lenort F., 1995, A Fast Discrete Fourier Transform with unequally-spaced frequencies, Journal of Theoretical and Applied Mechanics, 33, 1, 73-81

21. Liu T.I., Singonahalli J.H., Iyer N.R., 1996, Detection of roller bearing defects using expert system and fuzzy logic, Mechanical Systems and Signal Processing, 10, 5, 595-614

22. MA J., Li J.C., 1995, Detection of localised defects in rolling element bearing via composite hypothesis test, Mechanical Systems and Signal Processing, 9, 1, 63-75

23. Mann Jr L., Saxena A., Knapp G.M., 1995, Statistical-based or condition-based preventive maintenance, Journal of Quality in Maintenance Engineering, 1, 46-59

24. Mikhlin Y., Mytrokhin S., 2008, Non linear vibration modes of the tracked raod vehicle, Journal of Theoretical and Applied Mechanics, 46, 3, 581-596

25. Niu L., CaO H., He Z., Li Y., 2015, A systematic study of ball passing frequencies based on dynamic modeling of rolling ball bearings with localized surface defects, Journal of Sound and Vibration, 357, 207-232

26. Ou L., Yu D., YAng H., 2016, A new rolling bearing fault diagnosis method based on GFT impulse component extraction, Mechanical Systems and Signal Processing, 81, 162-182

27. Pachaud C., Salvetat R., Fray C., 1997, Crest factor and kurtosis contributions to identify defects inducing periodical impulsive forces, Mechanical Systems and Signal Processing, 11, 6, 903-916

28. Paulson E., 1947, A note on the efficiency of the Wald sequential test, The Annals of Mathematical Statistics, 18, 3, 447-450

29. Rai A., Upadhyay S.H., 2016, A review on signal processing techniques utilized in the fault diagnosis of rolling element bearings, Tribiology International, 96, 289-306

30. RENwiCK J.T., BABson P.E., 1985, 1985, Vibration analysis-a proven technique as a predictive maintenance tool, IEEE Transactions on Industry Applications, IA-21, 2

31. Rizos P.F., Aspragathos N., Dimarogonas A.D., 1990, Identification of crack location and magnitude in a cantilever beam from the vibration modes, Journal of Sound and Vibration, 138, 3, 381-388

32. Samanta B., Al-Balushi K.R., 2003, Artificial neural network based fault diagnosis for rolling element bearings using time-domain features, Mechanical Systems and Signal Processing, 17, 2, $317-328$

33. Schneeweiss H., 2005, Abraham Wald, Department of Statistics, University of Munich, Collaborative Research Center, 386, 439

34. Sheen Y.-T., 2004, A complex filter for vibration signal demodulation in bearing defect diagnosis, Journal of Sound and Vibration, 276, 105-119

35. SheEn Y.-T., 2008, An envelope detection method based on the first-vibration-mode of bearing vibration, Measurement, 41, 797-809

36. Sobel M., Wald A., 1949, A sequential decision procedure for choosing one of three hypotheses concerning the unknown mean of a normal distribution, The Annals of Mathematical Statistics, 20, 4, 502-522 
37. Stack J.R., Harley R.G., Habetler T.G., 2004, An amplitude modulation detector for fault diagnosis in rolling element bearings, IEEE Transactions on Industrial Electronics, 51, 5, 1097-1102

38. Tandon N., Choudhury A., 1999, A review of vibration and acoustic measurement methods for the detection of defects in rolling element bearings, Tribology International, 32, 8, 469-480

39. Tauqir A., Salam I., ul Haq A., Khan A.Q., 2000, Causes of fatigue failure in the main bearing of an aero-engine, Engineering Failure Analysis, 7, 127-144

40. Van M., Franciosa P., Ceglarek D., 2016, Rolling element bearing fault diagnosis using integrated nonlocal means de-noising with modified morphology filter operators, Mathematical Problems in Engineering, DOI: 10.1155/2016/9657285

41. WAld A., 1943, On the efficient design of statistical investigations, The Annals of Mathematical Statistics, 14, 2, 134-140

42. Wald A., 1945, Sequential tests of statistical hypotheses, The Annals of Mathematical Statistics, 16, $2,117-186$

43. Wald A., 1947, Sequential Analysis, John Wiley and Sons, New York

44. Wald A., 1949, Statistical decision functions, The Annals of Mathematical Statistics, 20, 2, $165-205$

45. Wald A., Wolfowitz J., 1943, An exact test for randomness in the non-parametric case based on serial correlation, The Annals of Mathematical Statistics, 14, 4, 378-388

46. WAld A., Wolfowitz J., 1948, Optimum character of the sequential probability ratio test, The Annals of Mathematical Statistics, 19, 3, 326-339

47. Wang H., Li R., Tang G., Yuan H., Zhao Q., Cao X., 2014, A compound fault diagnosis for rolling bearings method based on blind source separation and ensemble empirical mode decomposition, Journals Plos, 9, 10

48. Weiss L., 1956, On the uniqueness of Wald sequential tests, The Annals of Mathematical Statistics, 27, 4, 1178-1181

49. Wolfowitz J., 1949, On Wald's proof of the consistency of the maximum likelihood estimate, The Annals of Mathematical Statistics, 20, 4, 601-602

50. Yang H., Mathew J., MA L., 2005, Fault diagnosis of rolling element bearings using basis pursuit, Mechanical Systems and Signal Processing, 19, 341-356 